\title{
Chromospheric Evaporation in an M1.8 Flare Observed by the Extreme-ultraviolet Imaging Spectrometer on Hinode
}

\author{
G. A. Doschek \& H. P. Warren \\ Space Science Division, Naval Research Laboratory, Washington, DC 20375, USA \\ P. R. Young \\ College of Science, George Mason University, 4400 University Drive, Fairfax, VA 22030
}

\begin{abstract}
We discuss observations of chromospheric evaporation for a complex flare that occurred on 9 March 2012 near 03:30 UT obtained from the Extreme-ultraviolet Imaging Spectrometer (EIS) on the Hinode spacecraft. This was a multiple event with a strong energy input that reached the M1.8 class when observed by EIS. EIS was in raster mode and fortunately the slit was almost at the exact location of a significant energy input. Also, EIS obtained a full-CCD spectrum of the flare, i.e., the entire CCD was readout so that data were obtained for about the 500 lines identified in the EIS wavelength ranges. Chromospheric evaporation characterized by $150-200 \mathrm{~km} / \mathrm{s}$ upflows was observed in multiple locations in multi-million degree spectral lines of flare ions such as FexxII, FexxIII, and FexxIV, with simultaneous $20-60 \mathrm{~km} / \mathrm{s}$ upflows in million degree coronal lines from ions such as FexII-FeXVI. The behavior of cooler, transition region ions such as O vi, Fe viII, HeII, and Fex is more complex, but upflows were also observed in Fe VIII and Fex lines. At a point close to strong energy input in space and time, the flare ions Fe XXII, Fe XXIII, and Fe XXIV reveal an isothermal source with a temperature close to $14 \mathrm{MK}$ and no strong blueshifted components. At this location there is a strong downflow in cooler active region lines from ions such as Fe XIII and Fe XIV. We speculate that this downflow may be evidence of the downward shock produced by reconnection in the current sheet seen in MHD simulations. A sunquake also occurred near this location. Electron densities were obtained from density sensitive lines ratios from Fe XIII and Fe XIV. The results are combined with context Atmospheric Imaging Assembly (AIA) observations from the Solar Dynamics Observatory to obtain an overview of the flare and data are presented that can be used for predictive tests of models of chromospheric evaporation as envisaged in the Standard Flare Model.
\end{abstract}

Subject headings: Sun: flares, Sun: activity, Sun: UV radiation 


\section{INTRODUCTION}

Understanding energy release and transport in solar flares is one of the outstanding problems in contemporary solar physics. Solar flares have been observed from the radio to gamma ray wavelength ranges and solar flare models have been developed and continue to evolve and reach new levels of sophistication. The prevailing current view is embodied in the so-called Standard Flare Model, which has its origins in models such as proposed by Carmichael (1964), Sturrock (1968), Hirayama (1974), and Kopp \& Pneuman (1976). In the Standard Model, magnetic reconnection occurs in a current sheet in the corona, and results in plasma heating and particle acceleration. Heat conduction and high energy particles propagate along magnetic flux lines down into the chromosphere, heating the chromosphere and driving it upwards at multi-million degree temperatures into magnetic flux tubes closed by the reconnection process. The evaporated plasma explains the intensity and high electron density of soft X-ray emitting coronal loops. In addition, models of particle deposition into the chromosphere have shown that in some cases there is also a downward recoil produced by momentum conservation (e.g., Canfield et al. 1987; Fisher et al. 1985). Until recently, details of the evaporation process have been quite limited observationally.

The first spectroscopic observation of multi-million degree evaporation during flares was reported by Doschek et al. (1980) and Feldman et al. (1980). These authors noticed a blue spectral wing component present on the resonance lines of CaXIX near $3.17 \AA$ and Fe XXV near $1.85 \AA$. The spectra were from the SOLFLEX spectrometers on the Department of Defence (DoD) Pr8-1 spacecraft launched by the Air Force Space Test Program. Unfortunately these spectrometers had no spatial resolution and therefore it was not possible to observe evaporation in different spatial locations. Within two years more high resolution X-ray spectrometers were flown on the NASA Solar Maximum Mission spacecraft (e.g., Antonucci \& Dennis 1983) and the Japanese Hinotori spacecraft (e.g. Tanaka 1986). These missions confirmed the evaporation signature but also lacked high spatial resolution. Furthermore, the upflows were seen in only the highest temperature lines, such as the Caxix line, and were not observed in cooler lines due to poorer spectral resolution for the colder lines and lack of coverage at low temperatures. Thus, although the summed characteristics of evaporation were well-observed in the early 1980s for plasma at temperatures greater than about $10 \mathrm{MK}$, nothing was known about the evaporation process at different loop footpoints and at coronal temperatures near $1 \mathrm{MK}$.

The Bragg Crystal Spectrometer package flown in 1991 on the Japanese Yohkoh spacecraft (Culhane et al. 1991) greatly improved knowledge about the summed affects of evaporation on line profiles, but did not offer any information concerning the spatial distribution

or locations of evaporation. This changed with the launch of the Coronal Diagnostics Spec- 
trometer (CDS) (Harrison et al. 1995) on the ESA Solar \& Heliospheric Observatory. Several studies of evaporation at specific locations were made with CDS at footpoint locations in a line of Fe XIX at $592.23 \AA$ (i.e., Czaykowska et al. 2001; Teriaca et al. 2003; Brosius \& Phillips 2004, and Milligan et al. 2006a, 2006b).

The above data were interpreted with 1D hydrodynamic models (e.g., Nagai 1980; Cheng et al. 1983; Doschek et al. 1983; Nagai \& Emslie 1984; Emslie \& Nagai 1985) that eventually included loop tapering to simulate constricting flux tubes near footpoints, and adaptive grid meshes that allowed the chromosphere to move, although the transition region could not be resolved due to the spatially rapid changing temperature gradient that produces extremely small grid sizes. Energy and accelerated particles were introduced ad hoc anywhere desired in the coronal part of the loop. These early simulations predicted that at flare onset the entire line profile of all multi-million degree flare lines would be blueshifted. This was not observed in the summed flare data. The CDS spectra obtained later showed that these profiles were shifted, at least for Fe XIX, at flare footpoint regions. However, the lack of a shift at the very onset of flares was perplexing and contrary to model predictions (e.g., Doschek et al. 1986; Doschek \& Warren 2005).

A solution of the problem of the stationary multi-million degree line profiles at flare onset was first suggested by Hori et al. (1998). These authors showed with a numerical simulation that if the evaporation in a flux tube bundle took place sequentially in a number of unresolved smaller flux tubes within the larger magnetic envelop, then a stationary line profile could be be reproduced. This prediction was later tested with real flare data by Warren \& Doschek (2005) and found to reproduce very well the observed BCS line profiles from Yohkoh.

In summary, some of the Standard Flare Model predictions of the appearance of chromospheric evaporation are consistent with observations, but in general the total temperature coverage of evaporation at specific flare locations has still remained small. This has recently changed with the advent of the Extreme-ultraviolet Imaging Spectrometer (EIS) flown on the Japanese Hinode spacecraft. This spectrometer observes well spectral lines formed at 1 MK up to about $20 \mathrm{MK}$. It also observes a few lines of cooler transition region lines from ions such as Fe viII and HeII. Chromospheric evaporation at temperatures ranging from about $0.05 \mathrm{MK}$ up to $16 \mathrm{MK}$ was well observed in EIS spectra at loop footpoints for a C1.1 class impulsive flare by Milligan \& Dennis (2009). They found that even at the footpoints the line profiles of lines of multi-million degree Fe xxıII and Fe XXIV ions contained a stationary component, which they concluded is a disagreement with predictions of the Standard Model. However, more recent EIS spectra of a large number of flares have shown that in at least some cases all the high temperature emission is moving upwards at footpoints (Aoki \& Hara 
2012; Brosius 2013).

With the end of the extended solar minimum, EIS is observing more and more flares. It requires good fortune for the EIS slit to be at a significant flare location at a time when something significant might be learned. This has happened for an M1.8 flare that occurred on 9 March 2012. The EIS slit was virtually on top of a significant energy release site of the flare. Evaporative upflows were observed at many sites that we interpret as blended footpoint regions. We have found profiles for the multi-million degree Fe XXIII line near 263 $\AA$ that are completed shifted in wavelength towards the blue, almost completely stationary in wavelength, and in some cases are a mixture of shifted and unshifted components. In this paper we discuss these results and compare them with the results obtained by other authors using EIS flare data. Section 2 gives a brief summary of the EIS instrument, Section 3 describes the flare and available observations, results are given in Section 4 , and a discussion and summary are given in Section 5 .

\section{THE EIS ON HINODE AND DATA REDUCTION}

EIS is described by Culhane et al. (2007) and Korendyke et al. (2006). The instrument is a combination telescope and spectrometer. The telescope mirror and spectrometer grating are divided into two halves and each is coated with different Mo/Si multi-layers. Good sensitivity is achieved over two extreme-ultraviolet narrow wavebands: $170-210 \AA$ and $250-290$ $\AA$. Light from the telescope mirror is focused onto the entrance aperture of the spectrometer and passes through to a grating which diffracts and focuses the light onto two CCD detectors. There are four entrance apertures that can be selected: $1^{\prime \prime}$ slit, $2^{\prime \prime}$ slit, 40" slot, and $266^{\prime \prime}$ slot, all oriented in the north-south direction.

EIS can operate in raster mode, scanning from west to east, or in a sit and stare mode, with differential rotation included. At each position within a raster it is possible to readout the entire CCD (full-CCD) and obtain a complete spectrum for each wavelength band or alternatively one can select a small set of lines that fall in narrower spectral windows. The spatial resolution of EIS along the slit is nominally $2^{\prime \prime}\left(1^{\prime \prime}\right.$ per pixel) and the spectral dispersion is $0.0223 \AA$ per pixel. The instrumental full width at half maximum (FWHM) is about $0.056 \AA$. The actual spatial resolution is more like $3^{\prime \prime}$ due to the width of the point spread function.

The rastered flare data have been reduced using the standard EIS software data reduction package, i.e., the data are corrected for cosmic ray hits, warm pixels, detector bias, and dark current. We also corrected the wavelength positions for a variation of line position over 
the Hinode orbit due to temperature variations in the spectrometer and applied a correction for a slight tilt of the slit on the CCDs, which shifts wavelengths as a function of the north/south position of a pixel.

\section{OBSERVATIONS AND DATA REDUCTION}

The 9 March 2012 flare was a complex event that began in active region 11429 at N17, W12 near 03:24 UT. It was a multiple event with a first maximum at about 03:27 UT, where it reached class M1.8. After a small dip in intensity the X-ray flux continued to increase up to about M6.8. This increase occurred after the EIS slit had passed a portion of the active region that had begun to strongly flare, producing the additional intensity and an EIT wave. The Perfect Storm scenario is because the EIS slit was at or near a point of strong energy release that produced the first flare maximum, as indicated by the $193 \AA$ filter channel on the Atmospheric Imaging Assembly telescopes flown on the Solar Dynamics Observatory $(S D O)$. This channel showed a strong CCD saturation (bleeding in the north/south direction and highly localized in the east/west direction) very close to the location of the EIS slit. Unfortunately, after this energy pulse many of the AIA images are badly saturated for some time as the flare evolves. Neverthless, the first images are revealing regarding the locations of the strongest energy inputs. The location of the energy input observed by EIS is shown in Figure 1 along with GOES X-ray data for the flare. The vertical lines show the location of the EIS slit when a significant increase in flare intensity occurred. EIS scans from west to east (right to left in the Fe XIII line image shown).

The flare data were obtained by rastering the $2^{\prime \prime}$ slit from west to east across an active region in $2^{\prime \prime}$ steps with a 30s exposure time at each step. The raster covered $120^{\prime \prime}$ in the west-east direction (60 raster positions) and the slit length (north-south direction) was 160". Monochromatic images of solar regions were obtained by rastering the slit, integrating the spectra over wavelength, and then stacking the spectra side-by-side.

The particular active region raster study chosen for the 9 March active region was a full-CCD study, and therefore every spectral line observable by EIS was recorded. This not only allows examination of lines formed over a broad range of temperatures, but it also helps with saturation problems. For example, at the first pulse peak, the $192 \AA$ Fe xxIV line is saturated, but the substantially weaker $263 \AA$ FexxIII is not saturated. Thus, saturation effects are not as severe when weak lines from other ions or from the same ion can be used in place of saturated stronger ones.

A number of spectral lines were chosen for inspection and for analyzing evaporative 
upflows. They are listed in Table 1 with their wavelengths and temperatures of formation assuming ionization equilibrium. The temperatures are from the ion balance given in CHIANTI (Dere et al. 2009, A\&A, 498, 915).

Inspection of the line profiles shows that the lines of multi-million degree ions such as Fe XXIII have significant blueshifted components in some parts of the active region. This is where chromospheric evaporation is occurring along the same line-of-sight as plasma that has already reached a stationary state, i.e., no net Doppler speed. Since multiple upflowing sources are probably present along the same-line-of-sight, we simply divide the line profile into two Doppler speed intervals (see top left panel of Figure 6), rather than attempt a multiple fit with Gaussian line profiles. We then make images out of the centroid component and the two Doppler shifted asymmetric components. We don't know how many Gaussians to use. However, the lines from cooler coronal ions such as Fex - Fexvi do not show the broad blueshifted components and can therefore be fit with single Gaussians. Thus for each of these lines we obtain a total line intensity, a Doppler speed (from the centroid wavelength), and a line width (full width at half maximum, FWHM).

The FWHM is assumed to be due to instrumental broadening (known), thermal Doppler broadening, and excess broadening due to turbulence or just random non-thermal motions. The non-thermal broadening $V$ is measured assuming Gaussian distributions for all components, i.e.,

$$
F W H M=1.665 \times 10^{3} \frac{\lambda}{c} \sqrt{\frac{2 k T}{M}+V^{2}+W_{I}^{2}},
$$

where $\lambda$ is the wavelength (in $\AA$ in this paper), $c$ is the speed of light, $k$ is the Boltzmann constant, $T$ is the electron temperature, and $M$ is the ion mass. The instrumental width $W_{I}$ (also assumed Gaussian) is approximately $68 \mathrm{~m} \AA$ for the $2^{\prime \prime}$ slit, using the IDL routine eis_slit_width for the approximate location of the flare spectrum on the detector. Furthermore, if ionization equilibrium is assumed, there is the implicit assumption that the electron and ion temperatures are equal. This assumption is likely valid in the flare where the EIS observations are made, because electron densities in flares in the corona are known to be quite high (on the order of several times $10^{9}$ to several times $10^{11} \mathrm{~cm}^{-3}$. At these densities equilibration times between electrons and ions are very short, and ionization and recombination processes are very rapid for ions such as Fe XII.

Evaporation upflows or downflowing plasma speeds are determined from the wavelengths of the lines. Unfortunately EIS does not contain an absolute wavelength scale. We use rest wavelengths determined from off-limb observations by Warren et al. (2011) and assume that the uppermost and/or bottom part of the raster is essentially not moving along our line-of-sight. This region is well-outside of the flare region. 
Electron densities to be discussed below were determined from the Fexiı line ratio, 203.83/202.04 $\AA$ discussed by Young et al. (2009) and an Fe XIV ratio, 264.79/274.20 A. Using data in CHIANTI, the Fe XIII densities differ from densities obtained from an Fe XII line ratio used frequently in EIS papers (see Young et al. 2009) by about a factor of 2.5. This discrepancy is heading towards a resolution with revised atomic data for Fe XII (Del Zanna 2012) and also for Fe XIII. These data are not yet in CHIANTI but will appear in the next CHIANTI release. According to Young et al. (2012), with the old Fe XIII and Fe XII atomic data a $\log 10$ density of 8.6 determined from the Fe XIII ratio would be a $\log 10$ density of 8.9 using the Fe XII data. With the new atomic data, these ratios give $\log 10$ densities of 8.2 (Fe XIII) and 8.44 (Fe XII) and the error bars now overlap.

\section{RESULTS}

\subsection{Sites of Evaporating Plasma - Overview}

Figure 2 shows maps of the Doppler speed calculated from the centroid wavelengths of selected coronal spectral lines. In these images the rasters are put side by side, and are not separated by $2^{\prime \prime}$ as in Figure1. Figure1 is a correct image representation for comparison with AIA images, but Figure 2 shows the correct pixel numbers in the east-west direction and is therefore easier to relate to the EIS parameters. Another point to note is that the images are represented as they appear on the long wavelength EIS CCD. The short wavelength images have been shifted in the north-south direction by 17 pixels to be spatially aligned with the long wavelength images. The images concentrate on the flare region and are bounded by the yellow box in Figure 1. In the figure evaporating sites appear dark indicating negative Doppler speed. Note that all the lines show evaporation. For display the Fe xxiı Doppler map is made by subtracting the two blue-shifted component images from the centroid image after multiplying the blue-shifted intensities by 2. The sources of footpoints are not as clear as for the smaller event discussed by Milligan \& Dennis (2009).

Images of the flare in selected spectral lines are shown in Figure 3 through Figure 6 , shown with rasters spaced by $2^{\prime \prime}$ as in Figure 1. Transition region line images are shown in Figure 3, coronal line images are shown in Figure 4 and in the top panels of Figure 5 , The bottom panels of Figure 5 and Figure 6 show multi-million degree flare line images. In Figure 6] the images represent the emission bounded by the velocity intervals shown in the Fe Xxiı line profile panel. In Figure 5 and Figure [6] the mottling in the bright regions for the Fexv and FexxIV images is probably due to some saturated pixels.

Note that many of these figures show a small feature between 0 and 10 in X-pixels and 
15 an 20 in Y-pixels. This feature is brightest in transition region ions and is believed to be a fairly cold region although there is apparently a coronal component. Therefore, this emission in the bottom right panel of Figure 6 may not be due to Fe xxiII but may instead be due to weak unidentified lines in the blue wing of the FexxiII line. This is particularly true for the 260 - $470 \mathrm{~km} \mathrm{~s}^{-1}$ image. Weak unidentified lines far from the centroid of an emission line of interest can sometimes lead to confusion if the line of interest has a large blueshifted or redshifted component. See also Del Zanna et al. (2011) for a discussion of other weak lines that blend the short wavelength region of the Fe Xxiır line.

The short vertical lines in Figure 3 through Figure 5 indicate X-pixel locations (twice the actual pixel number) for which we show the evaporative speeds in detail. Figure 7 shows the Doppler speed plotted for four selected X-pixels as a function of Y-pixel for the entire raster in the north-south direction. The $\mathrm{X}$-pixel $=43$ was observed before the flare erupted in this region and shows no net strong flows. (The X-pixel $=43$ is not displayed in Figure 3 through Figure 5.) Similarly, no strong flows are seen for X-pixel $=27$, but evaporative upflows between 20 and $60 \mathrm{~km} \mathrm{~s}^{-1}$ are seen for X-pixels 12 and 19 (pixels 24 and 38 in Figure 3 through Figure 5) for lines of the ions indicated.

The variations of the upflow are shown in more detail in Figure 8 and Figure 9. In these figures the upflows are shown for eight consecutive X-pixels for four spectral lines, formed in typical active region loops. Note that the flows change significantly over a few arcsecond distances in both $\mathrm{X}$ and $\mathrm{Y}$.

The above results are concerned with coronal lines and the transition region ion, Fe VIII. The upflows seen in Fe XxIII and Fe XXIV are much greater. Sample Fe XXIII line profiles are shown in Figure 10. The inset image shows the locations of the profiles. FexxIII images with $2^{\prime \prime}$ intervals between rasters are shown in Figure 5 and Figure 6. These are the images to compare with AIA images. The numbers in parentheses in Figure 10 are the X-pixel and Y-pixel numbers, respectively. The intensity of the image at $(24,25)$ has been divided by two to fit within the plot. The peak intensities of the lines near X-pixel 24 show little or no blueshift and we assume that they are at or close to the rest wavelength. Most of the upflows are seen east of this position.

The eastward progression of the upflows can be seen in Figure 11. The Figure shows a selected path in the image and the upflow speeds obtained from the line centroids along this path, with position 0 near X-pixel $=12$ and position 10 near X-pixel position 27. The upflows here are not the blue-shifted wing of a profile, but are the shifts of the peak intensities of the profiles. Note that the coronal upflows are much less than the multi-million degree upflows. Transition region lines also show small upflows. 


\subsection{Sites of Evaporating Plasma - a Quantitative Example}

We provide some quantitative results for hydrodynamic modeling in Figure 12, Figure 13, and Figure 14 for one spectrum obtained at X-pixel $=12$. In these figures we give the Doppler speeds, intensities, and full widths at half maxima (FWHMs) for the lines in Table 1 for Fe viı,, Fe x, Fexiı, Fexiıı, Fe xiv, Fexv, Fexvi, and Fexxiı. The inset vertical line in Figure 12 shows the range of Y-pixels included in the plots. Also, in Figure 13 we give densities obtained from density sensitive intensity ratios of lines of Fe XIII and Fe XIV. The FexxiI lines in Figure 14 are mostly Gaussian in shape but shifted from their rest positions. However, in some cases there is a slight blueshifted wing. Therefore we have fit the FexxiII lines with both single Gaussians and with two Gaussians. All the data in Figure 14 are for the main Gaussian component. The image in Figure 14 shows the total FexxiII emission as well as the emission at wavelengths shortward of the rest wavelength, $263.76 \AA$. In the top left panel we show the results for both one and two Gaussian fits. They look quite similar. The one Gaussian fit gives slightly larger speeds as expected, and the two Gaussian fit shows more detail, also as expected. However, this detail may not be real. Finally, we have given FWHMs instead of non-thermal speeds in case a modeler does not wish to assume ionization equilibrium. Non-thermal speeds can be calculated using equation (1) and whatever temperature is assumed for the formation of the spectral line.

\subsection{The First Flare Peak}

We have noted that there was a large energy input near 03:26 UT that appears as a spatially-small, highly localized brightening in the 335 AIA channel. This occurred about 30s before the EIS slit reached this position, and therefore it is interesting to explore the dynamics of spectral lines near this location, as well as temperatures for the multi-million degree flare plasma. The coordinates of maximum Fe XXIII intensity are $\mathrm{X}=27, \mathrm{Y}=75$, at 03:26:27 UT.

Figure 15 shows Fe xxıI line intensities for X and Y pixels near $(27,75)$ and line profiles of Fexxiır, FexiıI, and Fexiv. Note that the Fexxiı emission is highly localized but that there is only a hint of a blue-wing on the line profile. The position is not a site of high velocity evaporation in the FexxiII line. Perhaps much more interesting are the red-wings that appear on the FeXIII and FexIV line profiles. Since these lines were fit by a single

Gaussian, the effect on the fit is to shift the centroids to the red, showing a downflow. This is seen in Figure 16] where the Doppler speeds are shown for four selected X-pixels around $(27,75)$. It is remarkable that the Doppler speeds of the two coronal lines track each other so well, even for small variations that might ordinarily be found to be noise. The two lines are 
not even from the same detector. The downflow is quite localized in position, and is striking for X-pixel 28. The EIS slit at $(27-28,75)$ is very close in space and time to a sunquake (Kosovichev 2012). In the next section we speculate on possible connections of the coronal downflows observed in this region and the sunquake.

At location $(27,75)$ five high temperature flare lines can be used to construct the loci plots in Figure 17 that give information on the differential emission measure of the source (e.g., see Warren \& Brooks 2009 for and explanation of loci plots). The figure shows loci plots for lines of FexviI, FexxiI, FexxiII, and Fexxiv. Again remarkably the highest temperature lines cross at virtually the same location, which indicates an isothermal source at about $14 \times 10^{6} \mathrm{~K}$. The Fe XVII line is formed at much lower temperatures and is formed outside the isothermal source.

\section{DISCUSSION}

The results in Section 4 show that the 9 March flare is quite complex in both space and time. EIS has observed upflow regions in unprecedented detail in both spatial and temperature coverage. In attempting to make sense of these observations it is important to keep in mind that the time resolution of EIS is low compared to the impulsive time scales of the beginning of this flare. The EIS exposure times are 30s, so that for example the eight $\mathrm{X}$-pixels for which data are shown in Figure 8 and Figure 9 cover four minutes of time in $\mathrm{X}$. However, all the data along $\mathrm{Y}$ are simultaneous.

An attempt can be made to understand the temporal/spatial evolution of the event by examining AIA filter data. However, we find that at flare onset some of the data is overexposed, and it is difficult to separate high temperature plasma from ions such as Fe XXIV from the cooler coronal emission that occurs in the same filter bandpass. Nevertheless, inspection of post-flare loops very late in the decay phase indicate that loops observed by EIS at flare onset were highly sheared relative to the neutral line (HMI data) and were basically in an east-west direction.

From inspection of all the figures in the previous section, we can conclude that:

1. The flare began before EIS observations began (see Figure 1). GOES data show that EIS began observing during the initial impulsive phase which was well-underway when EIS observing started.

2. Figure 2 shows that extensive outflow and downflow regions were observed between approximately $\mathrm{X}=0(03: 40: 42 \mathrm{UT})$ to $\mathrm{X}=30$ (03:24:52 UT) for coronal lines and the tran- 
sition region line of Fe VIII. Fe XXIII showed outflows almost over the entire raster between $\mathrm{X}=0$ and $\mathrm{X}=30$, due to the presence of blueshifted asymmetries on line profiles as well as blueshifts of the peak emission of the entire line profile.

3. In the coronal outflow regions the Fe XxiII emission can be modeled well with a single Gaussian, although a blueshifted component may also be present, possibly due to line-ofsight effects. The single Gaussians are Doppler shifted to the blue by varying amounts and the wavelengths (speeds) become larger (smaller) and approach the rest wavelength near X $=27$ (03:26:27 UT). This implies that between $\mathrm{X}=5$ (03:38:04 UT) and $\mathrm{X}=15$ (03:32:47 UT) we are observing a plethora of loop footpoint regions with varying degrees of outflows from two-arcsec pixel to pixel as is seen from Figure 8 and Figure 9. The fact that the FexxiII emission shows net outflows contradicts the results of Milligan \& Dennis (2009) who found a stationary component in addition to an outflow component at footpoint regions and were puzzled by it since models predict net shifts there. However, the implication is that the actual strengths of outflows and the temperatures at which they occur depend critically on the energy input into the chromosphere from the corona, and perhaps also on the nature of the input, i.e., conduction fronts or energetic particles. Observations can easily be complicated by line-of-sight effects or unresolved footpoint regions. Milligan \& Dennis (2009) found downflows in the footpoint regions for coronal and transition region lines, which also contradicts the present results. The 9 March flare is a considerably more energetic event than the event observed by Milligan \& Dennis (2009), which probably explains the differences in results. We note that Kunichika Aoki \& Hirohisa Hara (oral paper, Hinode 6 meeting, St. Andrews, Scotland 2012) also reported entire shifts of the Fe XxiII line centroid at footpoint regions from EIS picket-fence raster flare studies. Similarly, Brosius (2013) obtained entire shifts of the FexxIII profile from EIS spectra obtained from a rapid cadence stare mode observation for a C1 flare (See also Del Zanna et al. 2011). Previously, Watanabe et al. (2010) reported on variably shaped Fe XXIII profiles from EIS spectra in a flare and Young, Doschek, \& Warren (2013) also find Fexxiı profiles with intense blueshifted components. The conclusion is that highly variable results at footpoint regions can be expected depending on energy input and probably also the impulsiveness of the flare. We have given results (Figure 12, Figure 13, and Figure 14) for one X position for a quantitative modeling exercise.

4. The region near $(27,75)$ is special because of the strong energy input that occurred there and produced the first flare peak, and the presence of a sunquake close to the EIS slit position. The redshifted wing asymmetries that occur on the coronal lines are unique to the region around $(27,75)$. The profiles of the multi-million degree flare lines are well-fit by single Gaussians near or at the rest wavelengths. In the Standard Flare Model, this is the expectation for the emission near the tops of loops after it has come to rest after evaporation. Such plasma could exist at this loop location because the flare began about 
a minute prior to EIS observations. In this time plasma could have reached loop tops and produced stationary emission. Nevertheless, this emission appears quite impulsive in time, i.e., it appears as a relatively small spot seen in Figure 1 (the EIS slit goes through it) around positions $(2 \times 27,75)$. If the energy impulse at this time is due to a reconnection event above the closed loop tops as in the Standard Model, then the downflowing coronal plasma might be due to the downward shock wave produced in the reconnection. If so, to our knowledge this is the first observation of the downward propagating shock. However, this is still simply a speculation. The downward flowing coronal plasma might also somehow be linked to the sunquake, but unfortunately we have only snapshot observations where higher spatial resolution is also needed to resolve individual structures.

In summary, the EIS observations in this paper, coupled with results in other papers, reveal multiple complex footpoint regions that give rise to different evaporation dynamics over small spatial scales. This indicates a complex energy input from the corona, suggesting modelling to see if the EIS results can be reproduced by different energy inputs, e.g., electron beams, conduction fronts, with different values for total energy input and different temporal behavior, etc.

Tthe EIS observations make a strong case for the Extreme Ultra-Violet Spectroscopic Telescope (EUVST) proposed for the Solar-C mission (see Doschek et al., Solar-C white paper, Heliophysics Decadal Survey). Proper slit observations for flares requires higher spatial resolution, much higher temporal resolution, and greater temperature coverage in the transition region and chromosphere. Also desirable are filter telescope images that do not saturate at flare onset and that have clear bandpasses for picking out the multi-million degree flare emission from coronal and transition region emission.

Hinode is a Japanese mission developed and launched by ISAS/JAXA, collaborating with NAOJ as domestic partner, and NASA (USA) and STFC (UK) as international partners. Scientific operation of the Hinode mission is conducted by the Hinode science team organized at ISAS/JAXA. This team mainly consists of scientists from institutes in the partner countries. Support for the post-launch operation is provided by JAXA and NAOJ, STFC, NASA, ESA (European Space Agency), and NSC (Norway). We are grateful to the Hinode team for all their efforts in the design, build, and operation of the mission.

The authors acknowledge support from the NASA Hinode program and from ONR/NRL 6.1 basic research funds. 


\section{REFERENCES}

Antonucci, E., \& Dennis, B. R. 1983, Sol. Phys., 86, 67

Aoki, K. \& Hara, H. 2012, oral paper at the Hinode 6 meeting in St. Andrews, Scotland

Brosius, J. W. 2013, ApJ, in press for Vol. 762, 10 January 2013

Brosius, J. W., \& Phillips, K. J. H. 2004, ApJ, 613, 580

Canfield, R. C., Metcalf, T. R., Strong, K. T., \& Zarro, D. M. 1987, Nature, 326, 165

Carmichael, H. 1964, in AAS-NASA Symposium on the Physics of Solar Flares, ed. W. N. Hess (NASA SP-50), 451

Cheng, C.-C., Oran, E. S., Doschek, G. A., Boris, J. P., \& Mariska, J. T. 1983,ApJ, 265, 1090

Culhane, J. L., et al. 1991, Sol. Phys., 136, 89

Culhane, J. L., et al. 2007, Sol. Phys., 243, 19

Czaykowska, A., Alexander, D., \& De Pontieu, B. 2001, ApJ, 552, 849

Del Zanna, G. 2012, presentation at the Spectroscopy of the Dynamics Sun meeting, Univ. College London, 18-20 April, UK.

Del Zanna, G., Mitra-Kraev, U., Bradshaw, S. J., Mason, H. E., \& Asai, A. 2011, Astronomy \& Astrophysics, 526, A1

Dere, K. P., et al. 2009, Astronomy \& Astrophysics, 498, 915

Doschek, G. A., Feldman, U., Kreplin, R. W., \& Cohen, L. 1980, ApJ, 239, 725

Doschek, G. A., Cheng, C.-C., Oran, E. S., Boris, J. P., \& Mariska, J. T. 1983, ApJ, 265, 1103

Doschek, G. A., et al. 1986, chap. 4 in Energetic Phenomena on the Sun, proceedings from the Solar Maximum Mission Workshop, ed. M. Kundu \& B. Woodgate, NASA Conference Publication 2439

Doschek, G. A., \& Warren, H. P. 2005, ApJ, 629, 1150

Emslie, A. G., \& Nagai, F. 1985, ApJ, 288, 779

Feldman, U., Doschek, G. A., Kreplin, R. W., \& Mariska, J. T. 1980, ApJ, 241, 1175

Fisher, G. H., Canfield, R. C., \& McClymont, A. N. 1985, ApJ, 289, 414

Harrison, R. A., et al. 1995, Sol. Phys., 162, 233

Hirayama, T. 1974, Sol. Phys., 34, 323

Hori, K., Yokoyama, T., Kosugi, T., \& Shibata, K. 1998, ApJ, 500, 492 
Kopp, R. A., \& Pneuman, G. W. 1976, Sol. Phys., 50, 85

Korendyke, C. M., et al. 2006, Applied Optics, vol. 45, Issue 34, 8674

Kosovichev, A. G. 2012, 220th Meeting of the AAS, Anchorage, Alaska, 11 June 2012.

Milligan, R. O., Gallagher, P. T., Mathioudakis, M., \& Keenan, F. P. 2006a, ApJ, 642, L169

Milligan, R. O., et al. 2006b, ApJ, 638, L117

Milligan, R. O., \& Dennis, B. R. 2009, ApJ, 699,968

Nagai, F. 1980, Sol. Phys., 68, 351

Nagai, F., \& Emslie, A. G. 1984, ApJ, 279, 896

Sturrock, P. A. 1968, in Structure \& Development of Solar Active Regions, ed. K. Kiepenheuer (Paris: IAU), 471

Tanaka, K. 1986, Publ. Astron. Soc. Japan, 38, 225

Teriaca, L., Falchi, A., Cauzzi, G., Falciani, R., Smaldone, L. A., \& Andretta, V. 2003, ApJ, 588,596

Warren, H. P., \& Brooks, D. H. 2009, ApJ, 700, 762

Warren, H. P., \& Doschek, G. A. 2005, ApJ, 618, L157

Warren, H. P., Ugarte-Urra, I., Young, P. R., \& Stenborg, G. 2011, ApJ, 727, 58

Watanabe, T, Hara, H., Sterling, A. C., \& Harra, L. K. 2010, ApJ, 719, 213

Young, P. R., Del Zanna, G., Dere, K. P., Landi, E. 2012, \& Mason, H. E. 2012, presented at the Eighth International Conference on Atomic and Molucular Data and Their Applications, National Institute of Standards and Technology, Gaithersburg, MD, 30 September - 4 October.

Young, P. R., Doschek, G. A., \& Warren, H. P. 2013, ApJ, in preparation

Young, P. R., Watanabe, T., Hara, H., \& Mariska, J. T. 2009, Astronomy \& Astrophysics, 495, 587

This preprint was prepared with the AAS LATEX macros v5.2. 
Table 1: Spectral Lines, Wavelengths, and Temperatures of Formation

\begin{tabular}{lll}
\hline \hline Ion & Wavelength $(\AA)$ & Temperature $(\mathrm{K})$ \\
\hline He II & 256.32 & $5.0 \times 10^{4}$ \\
O v & 248.46 & $2.2 \times 10^{5}$ \\
O vi & 184.12 & $2.8 \times 10^{5}$ \\
Fe VIII & 185.211 & $4.0 \times 10^{5}$ \\
Fe X & 184.534 & $1.1 \times 10^{6}$ \\
Fe XII & 192.394 & $1.6 \times 10^{6}$ \\
Fe XII & 195.119 & $1.6 \times 10^{6}$ \\
Fe XIII & 202.047 & $1.8 \times 10^{6}$ \\
Fe XIII & 203.824 & $1.8 \times 10^{6}$ \\
Fe XIV & 264.783 & $2.0 \times 10^{6}$ \\
Fe XIV & 274.202 & $2.0 \times 10^{6}$ \\
Fe XV & 284.160 & $2.2 \times 10^{6}$ \\
Fe XVI & 262.967 & $2.8 \times 10^{6}$ \\
Fe XVII & 254.88 & $4 \times 10^{6}$ \\
Fe XXII & 253.17 & $1.3 \times 10^{7}$ \\
Fe XXIII & 263.76 & $1.4 \times 10^{7}$ \\
Fe XXIV & 255.10 & $1.8 \times 10^{7}$ \\
\hline
\end{tabular}




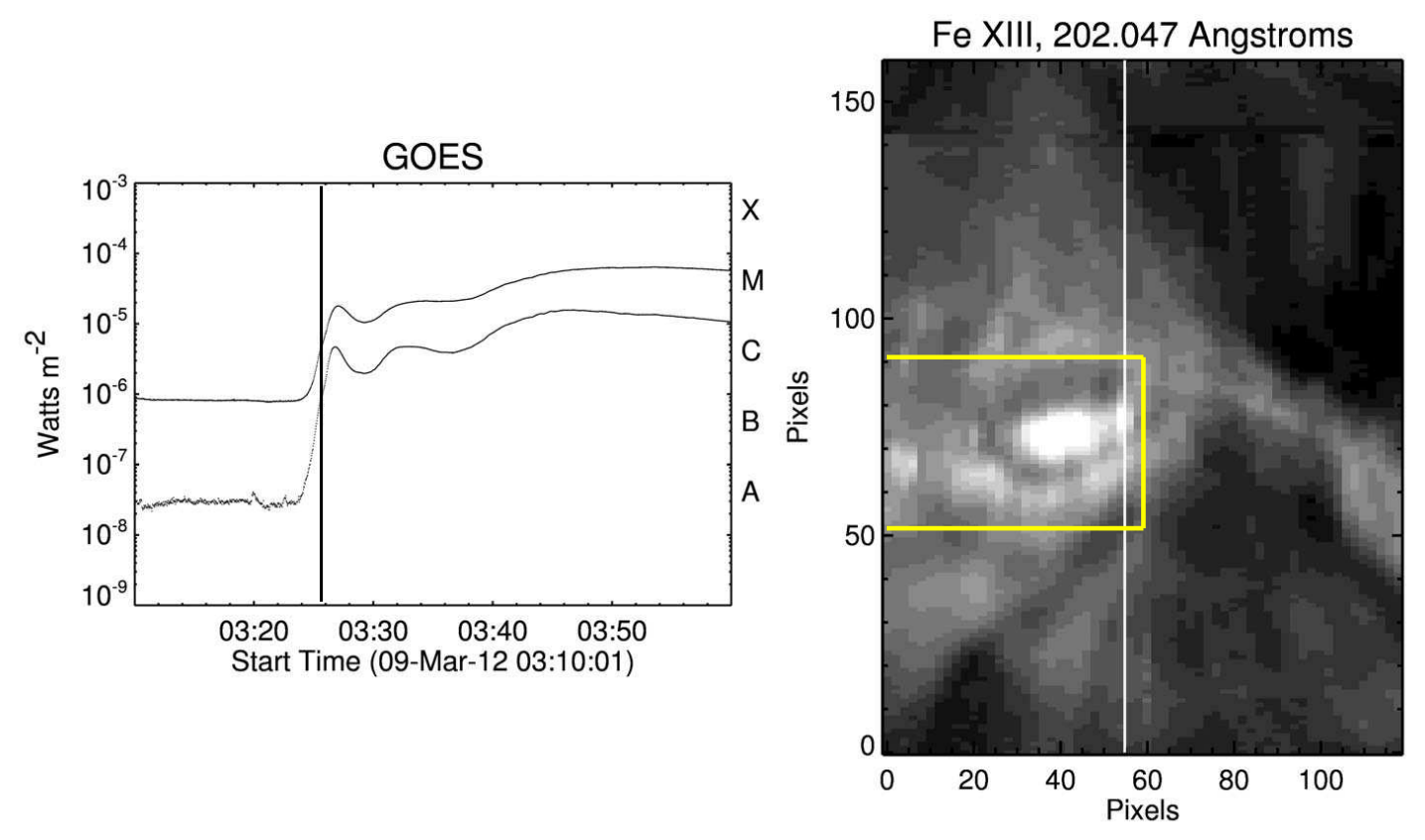

Fig. 1. - The location of the EIS slit (vertical lines) in an EIS image of the flare and relative to X-ray emission recorded by GOES. EIS scans from west to east (right to left in the image). 

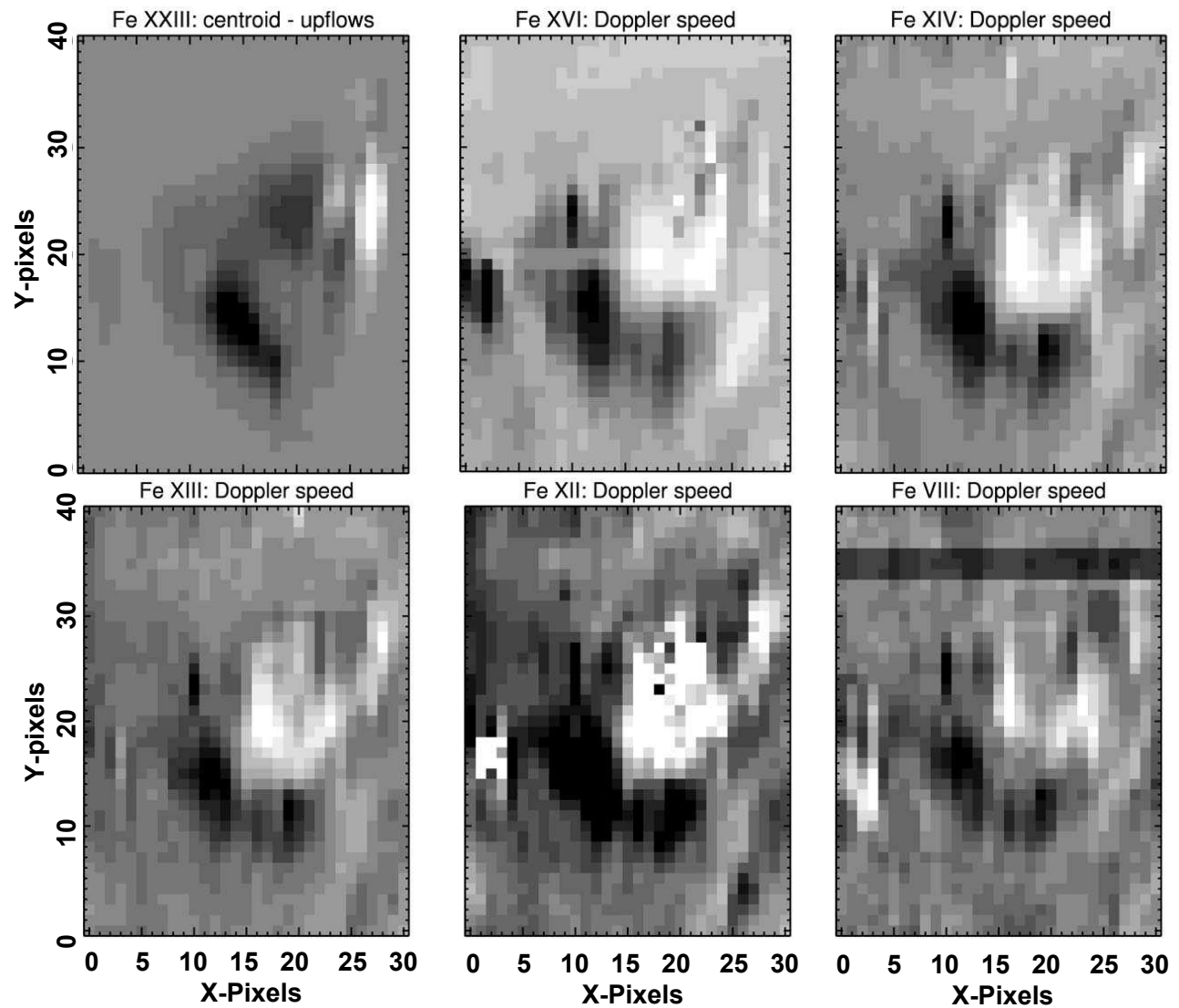

Fe VIII: Doppler speed

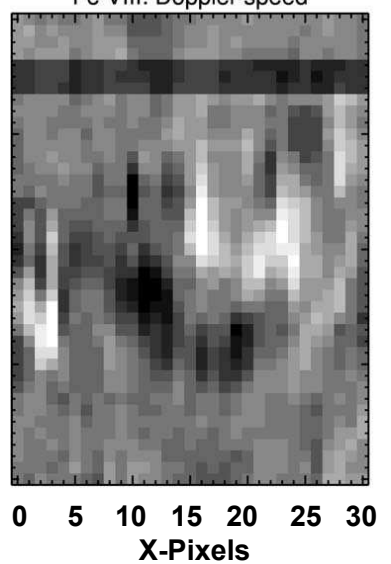

Fig. 2.- Centroid maps of selected spectral lines. The maps are bounded by the yellow box in Figure 1. Dark indicates an upflow or evaporating plasma. 

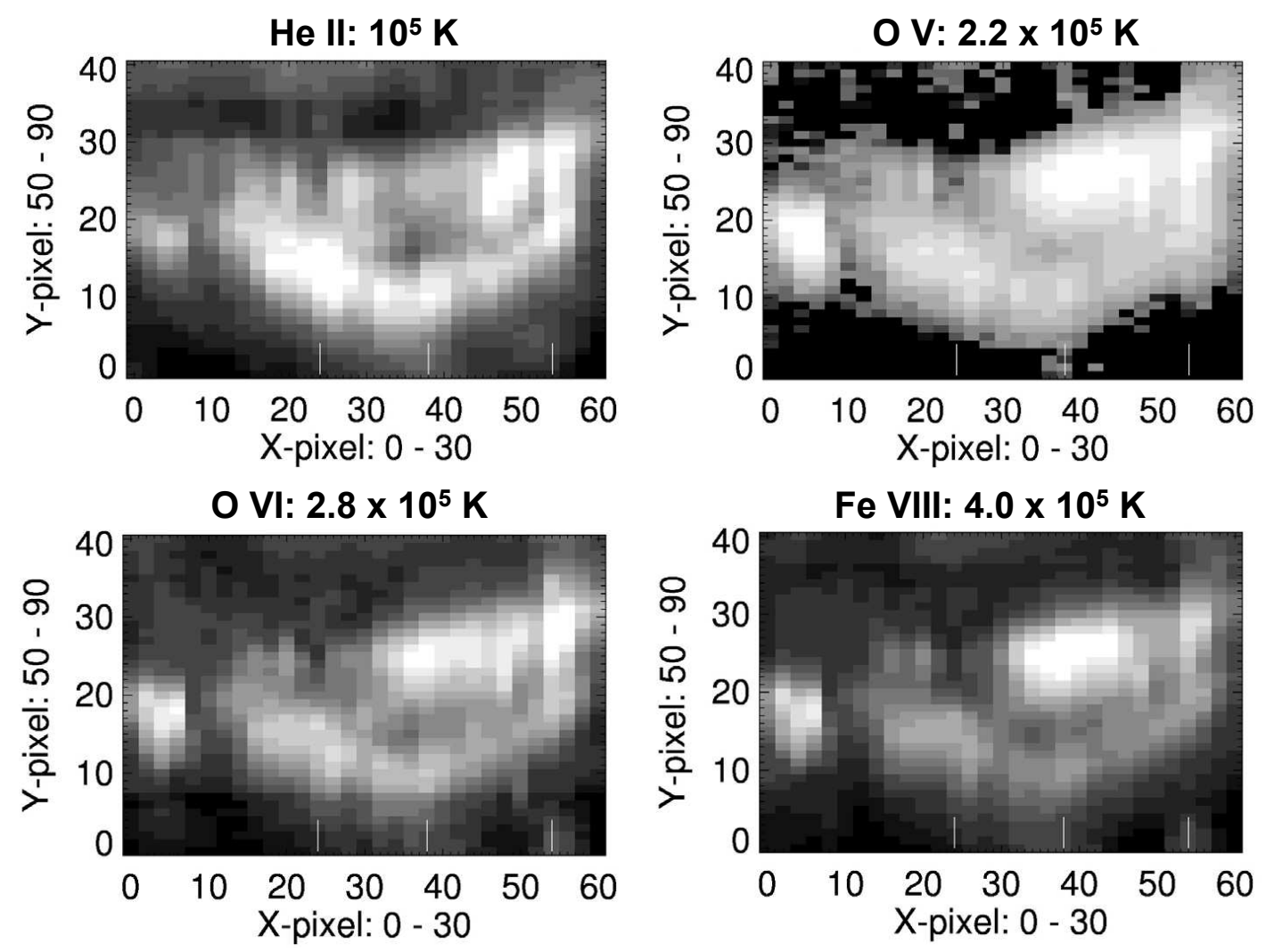

Fig. 3.- Transition region line images of the flare region bounded by the yellow box in Figure 1, The short vertical lines refer to X-pixels for which detailed evaporation speeds are shown in Figure 7 . 
Fe X: $1.1 \times 10^{6} \mathrm{~K}$

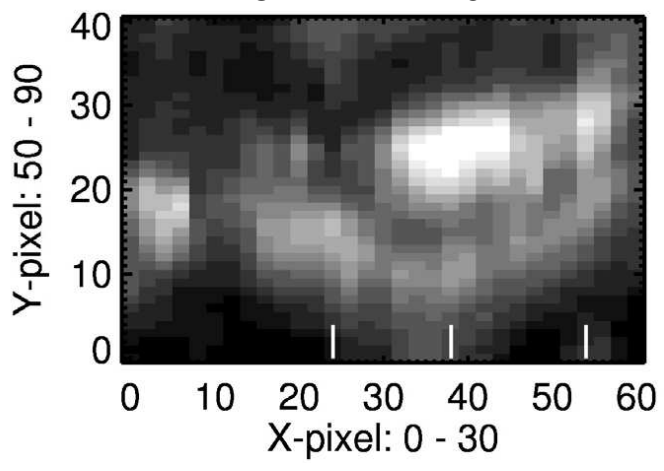

Fe XIII: $1.5 \times 10^{6} \mathrm{~K}$

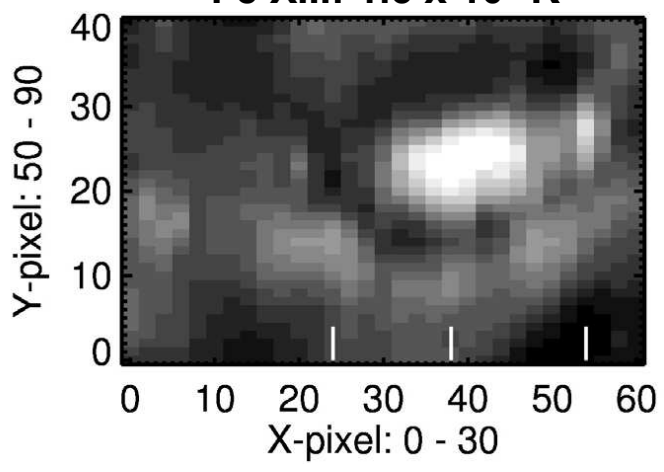

Fe XII: $1.5 \times 10^{6} \mathrm{~K}$

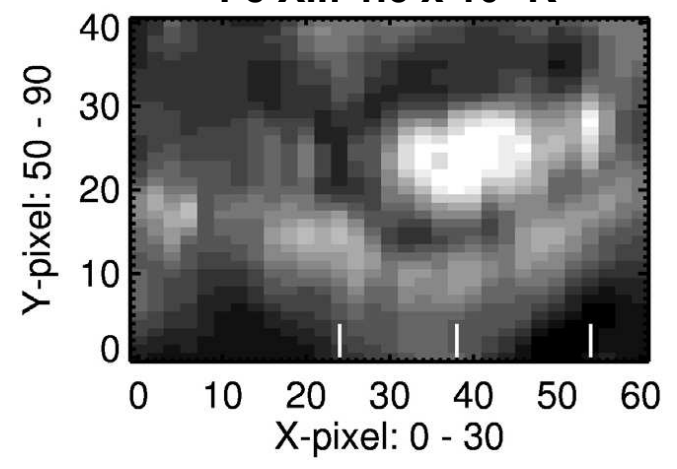

Fe XIV: $2.0 \times 10^{6} \mathrm{~K}$

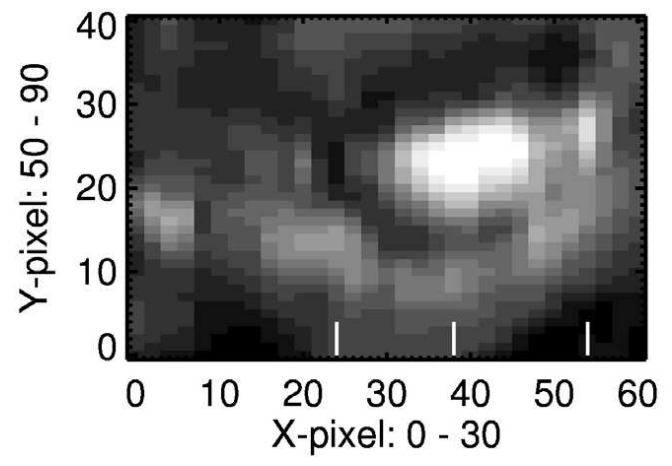

Fig. 4.- Coronal line images of the flare similar to Figure 3 , 

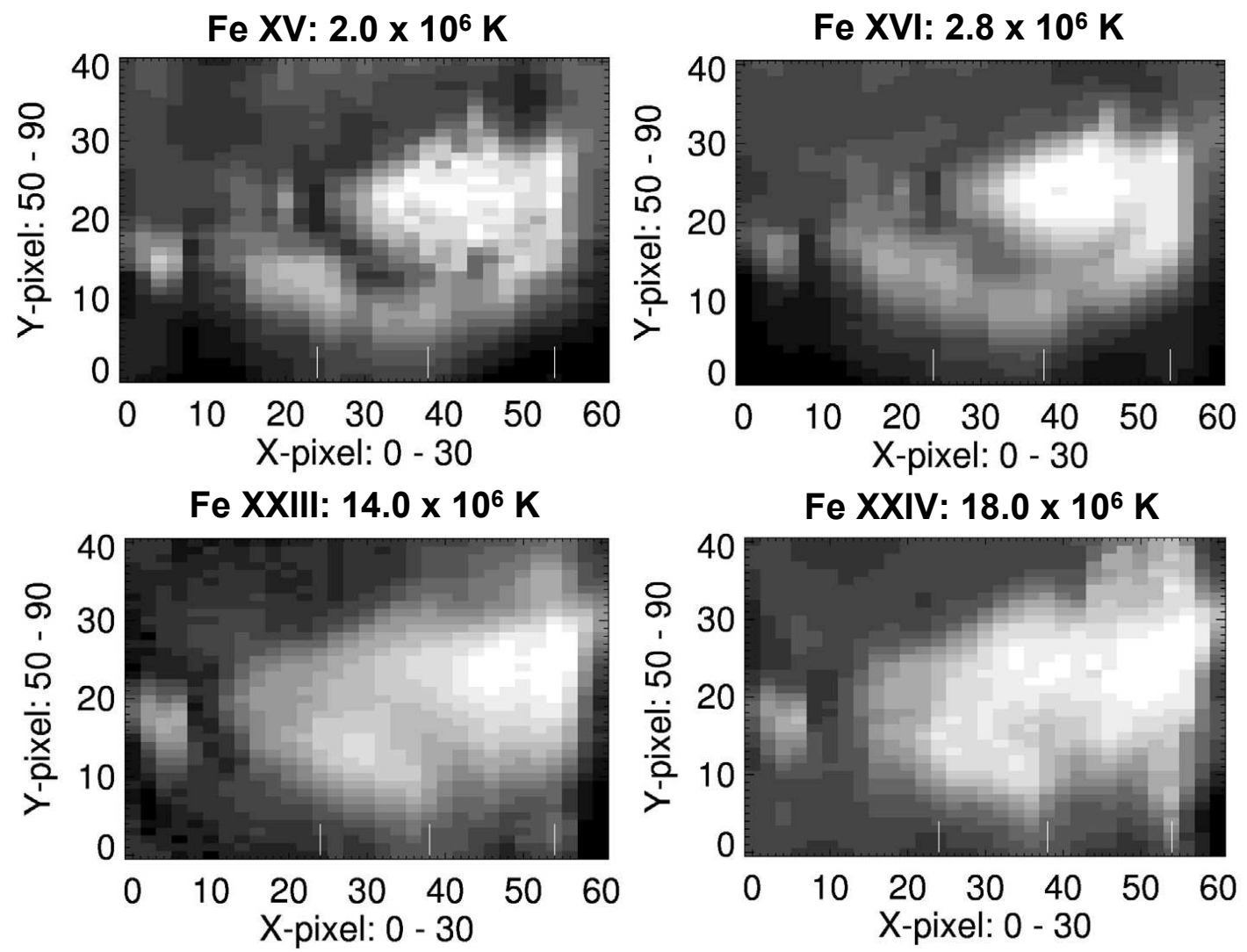

Fig. 5.- Coronal line and multi-million degree flare line images of the flare similar to Figure 3 , 

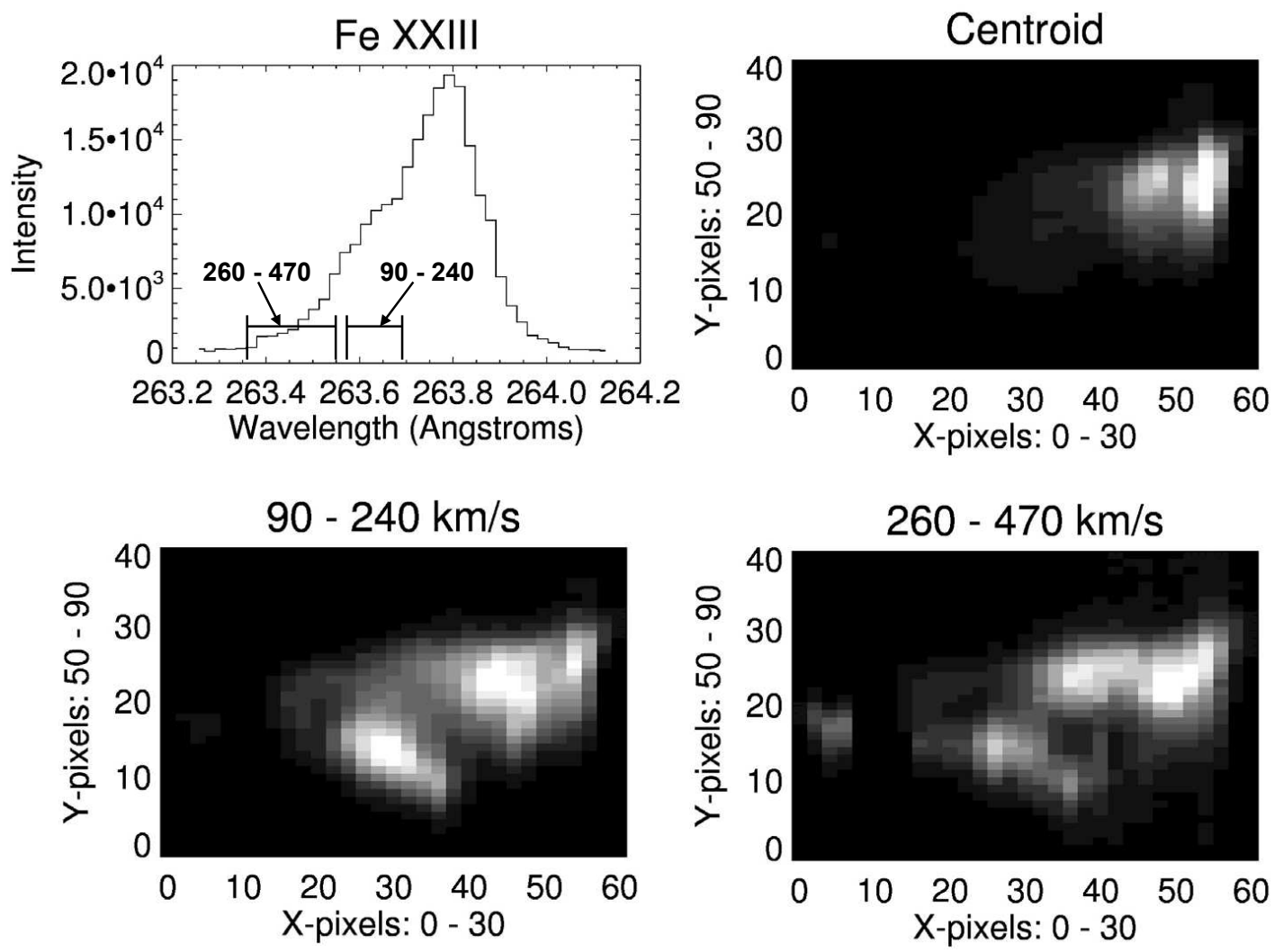

Fig. 6. - Fe Xxiır images of the flare in the velocity intervals $90-240$ and $260-470 \mathrm{~km} \mathrm{~s}^{-1}$ defined by the FexxiII line profile in the top left panel. 

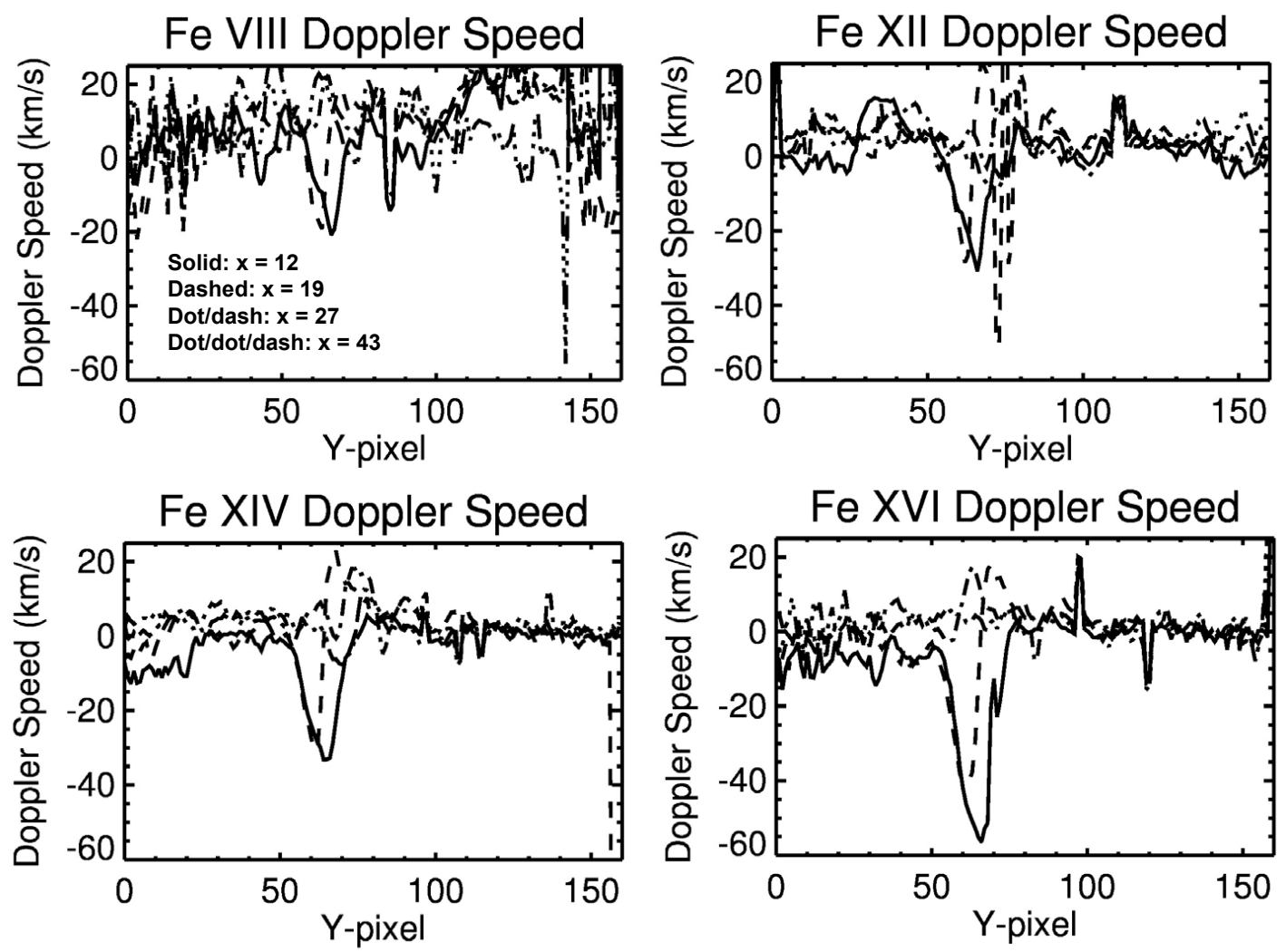

Fig. 7.- Evaporative speeds for lines of the ions indicated for 4 selected X-pixels. Negative speeds are upflows towards the observer. 

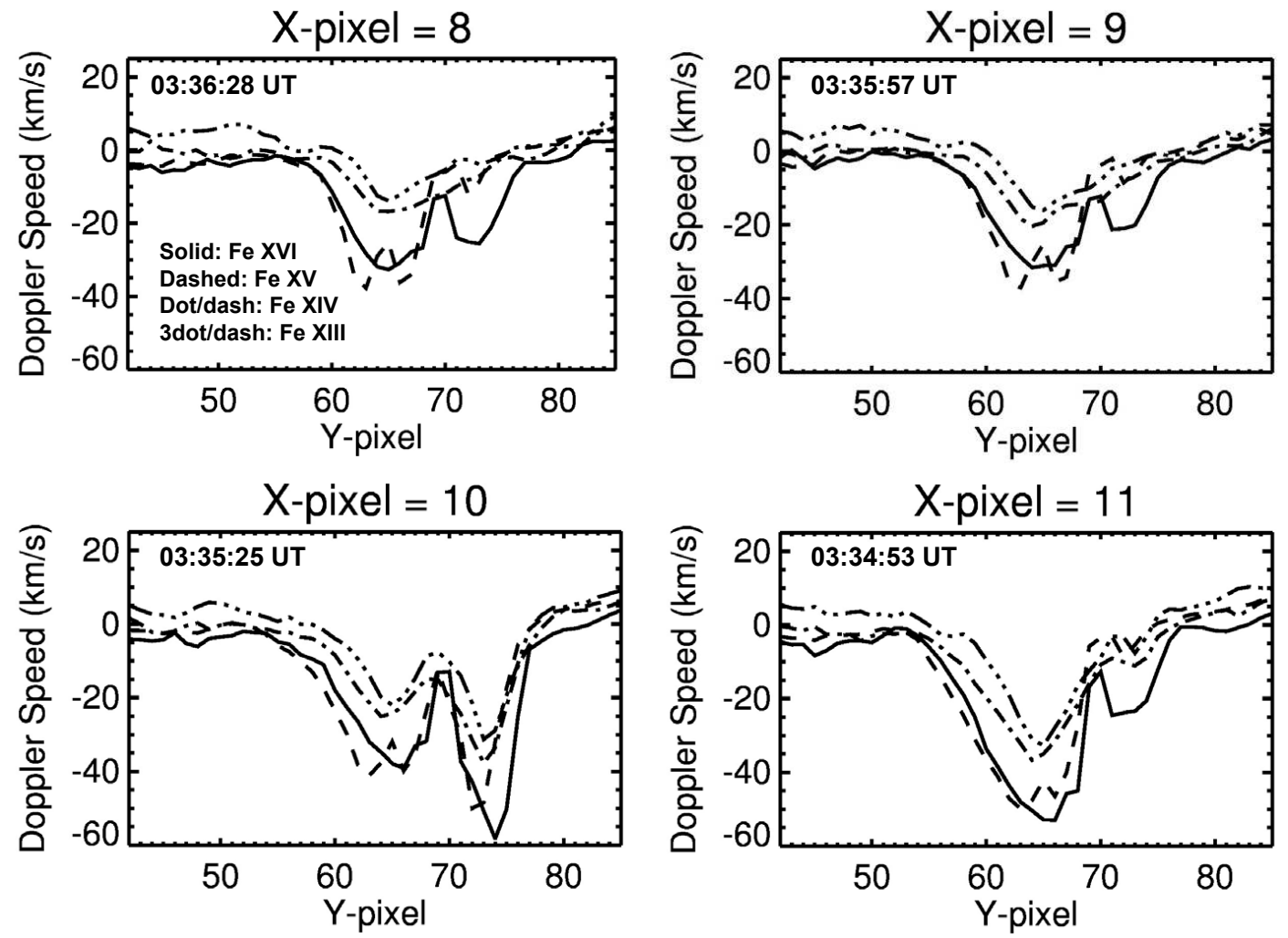

Fig. 8.- Details of upflows for selected X-pixels in the lines of FexIII, Fe XIV, Fe XV and Fe XVI. 

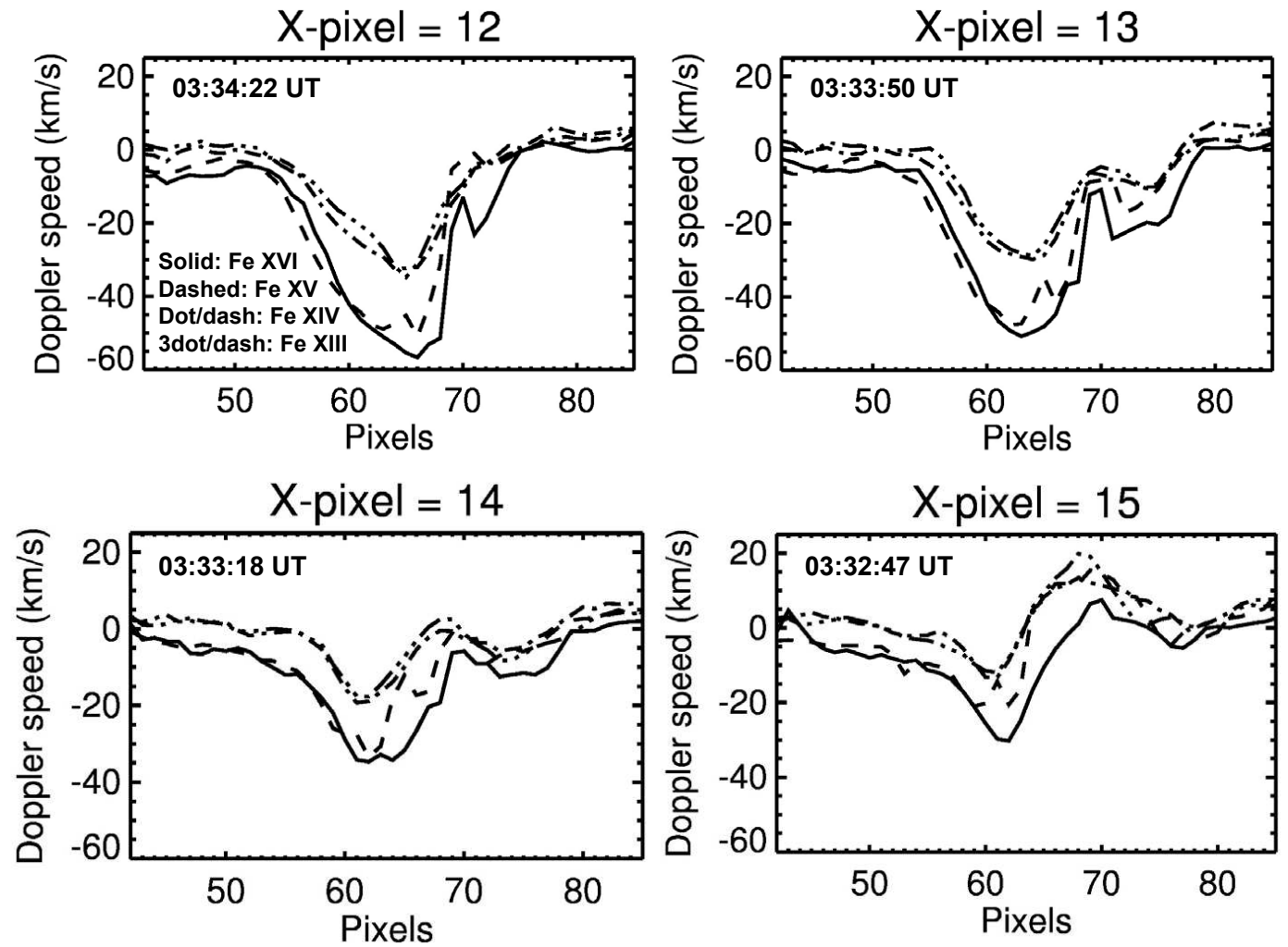

Fig. 9.- Details of upflows for selected X-pixels in the lines of FexIII, Fe XIV, FeXV and FeXvI. 
Fe XXIII, 263.76 Angstroms

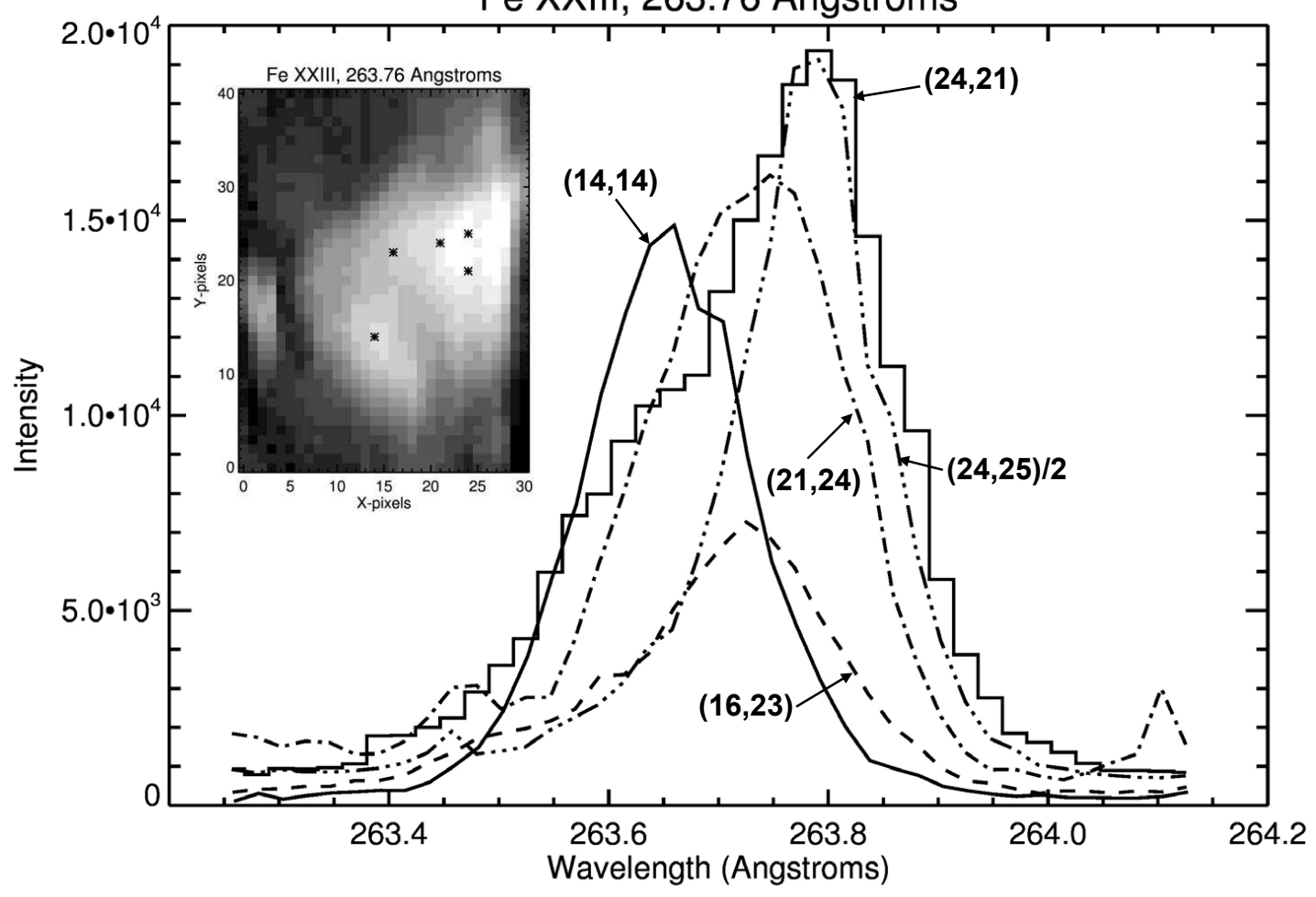

Fig. 10.- Line profiles of the FeXXIII line at selected X and Y pixels. The numbers in parentheses are the $\mathrm{X}$ and $\mathrm{Y}$ pixels, respectively. The intensity of the $(24,25)$ profile has been divided by 2 to fit onto the plot. The inset image shows the pixel positions (see also the $2^{\prime \prime}$ raster position images in Figure 5 and Figure 6 for comparison with AIA images.) 


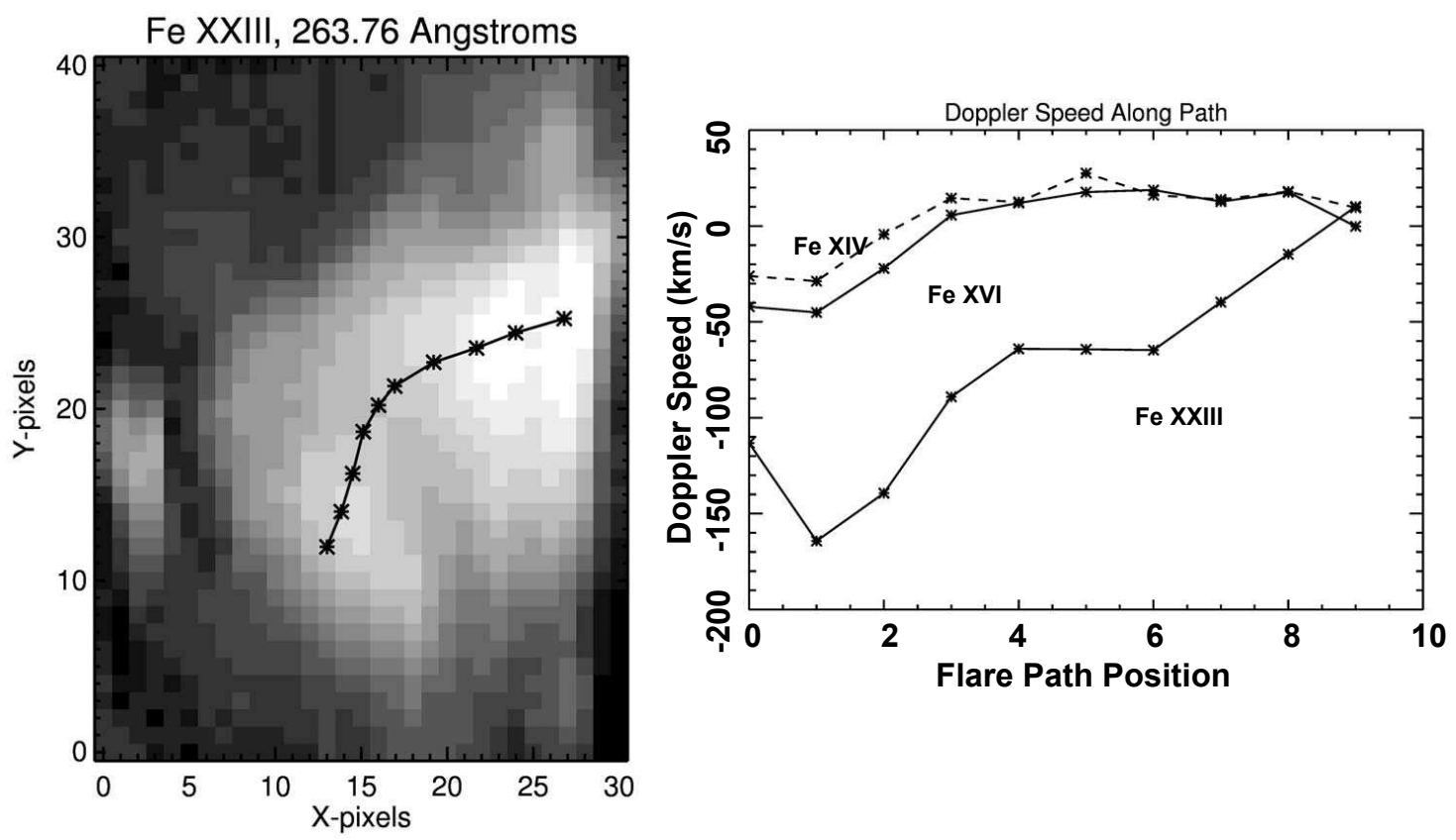

Fig. 11. - The Doppler speeds of the lines of the three ions shown as a function of position along the path. Position 0 is near X-pixel $=12$. 

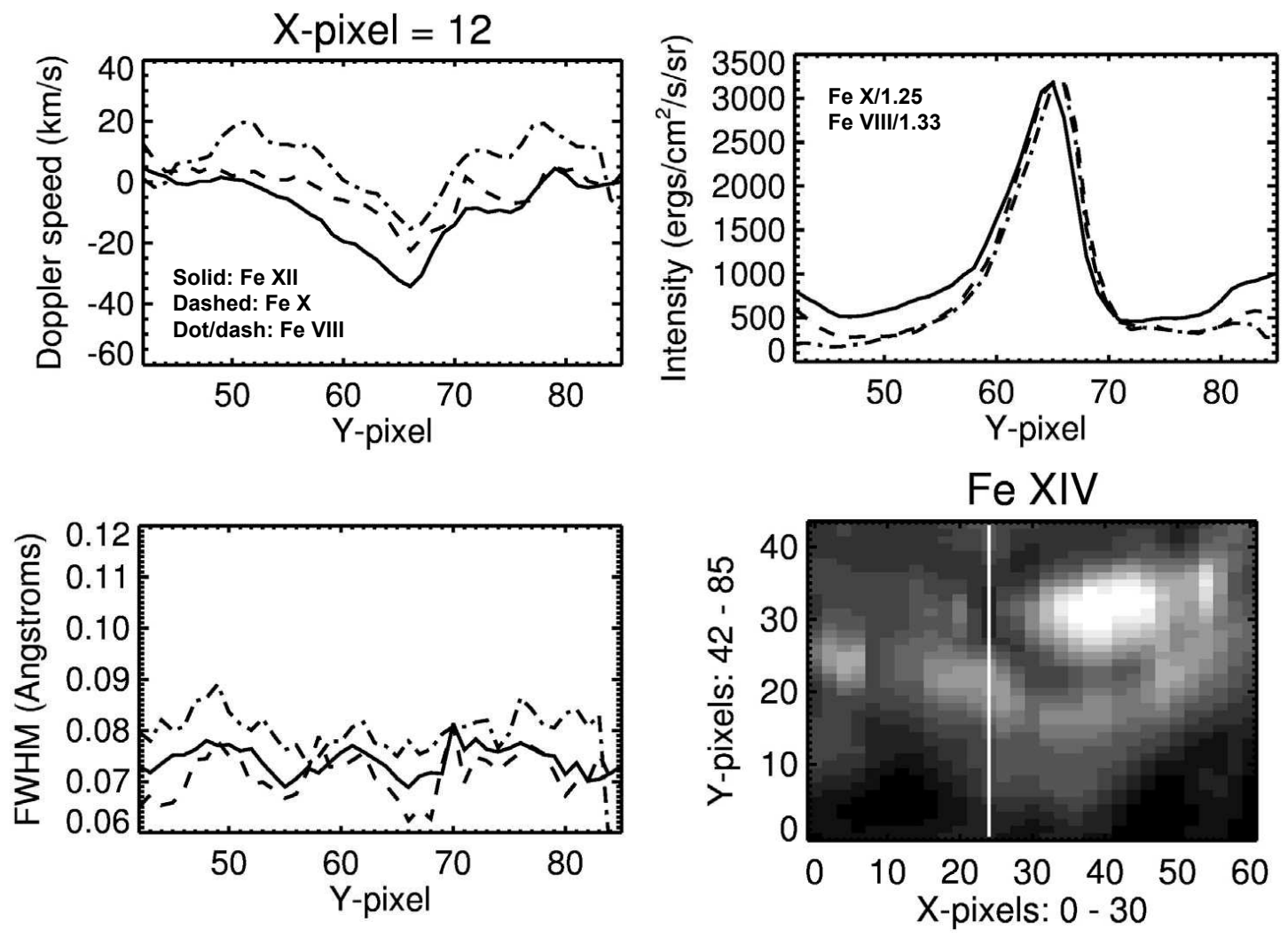

Fig. 12.- Doppler speed, intensity, and FWHM for the upflow region at X-pixel $=12$. The intensities of the Fe VIII and Fex lines are normalized to the intensity of the Fe XII line. The vertical line in the Fe XIV image shows the range of Y-pixels included in the plots. 

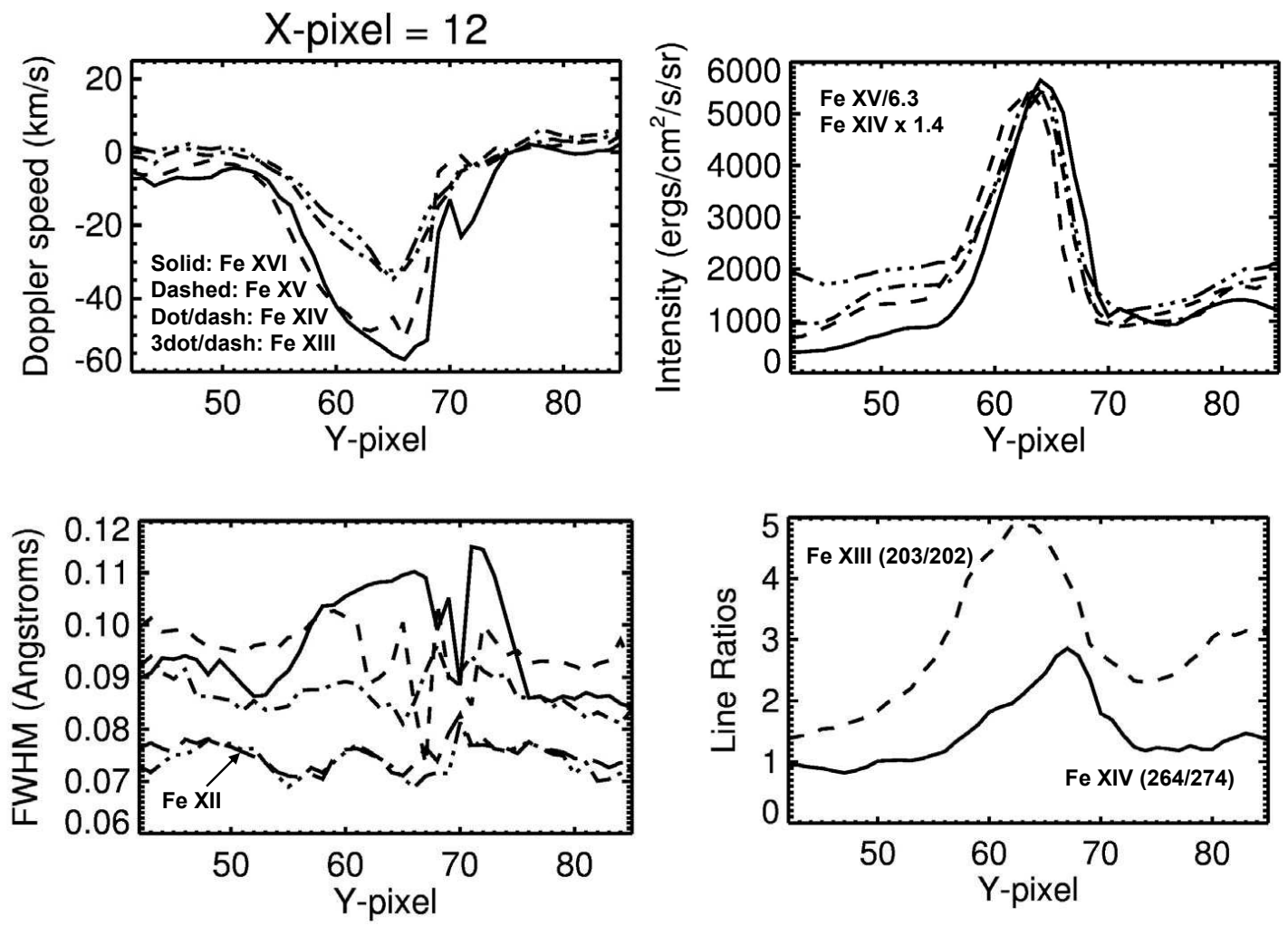

Fig. 13.- Similar to Figure 12 for lines of Fe XIII - Fe XVI. The normalized intensities are indicated. The lower right plot gives density sensitive line ratios for Fe XIII and Fe XIV lines. 

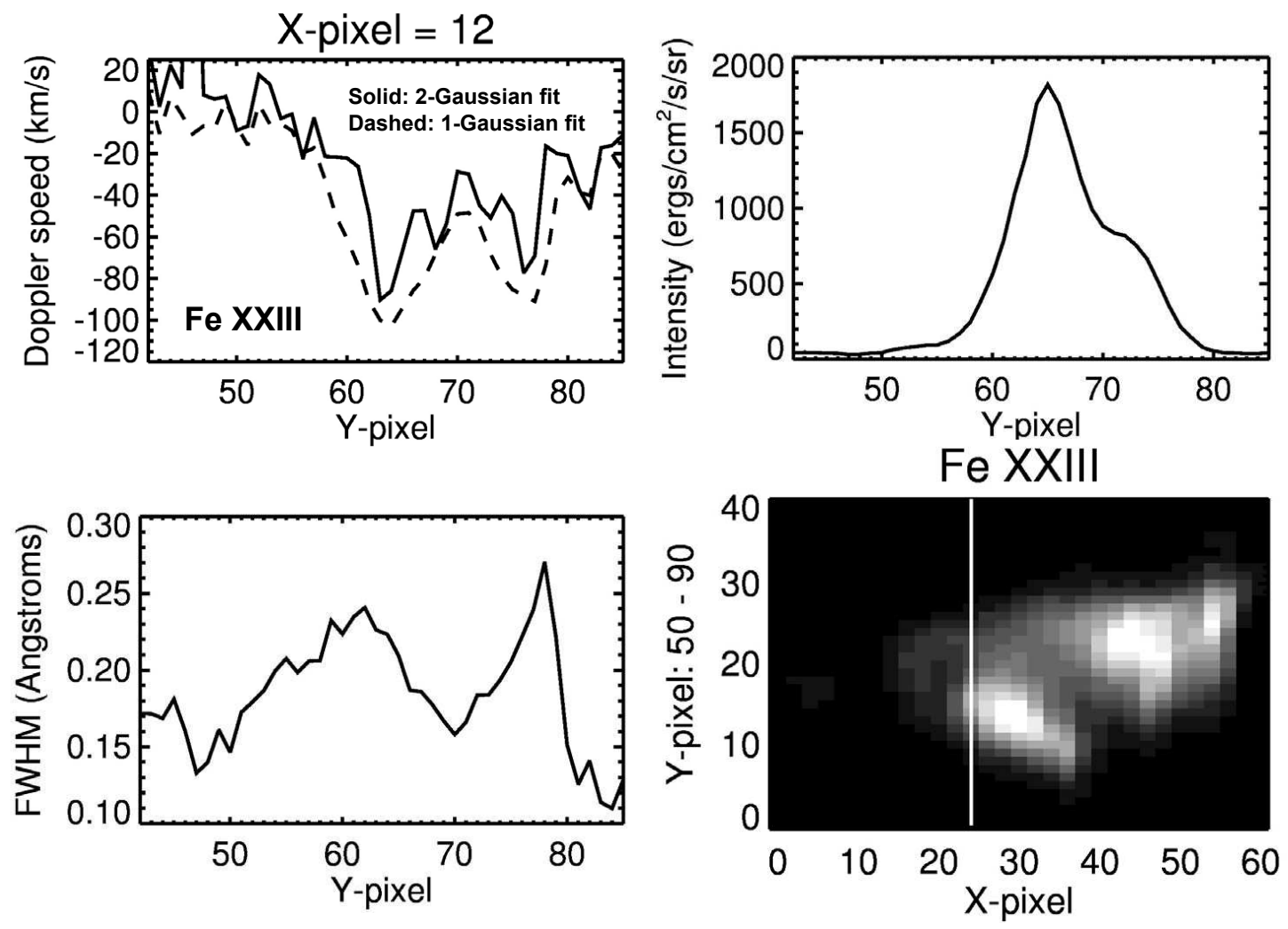

Fig. 14.- Similar to Figure 12 for the Fe XXIII line. 
Fe XXIII

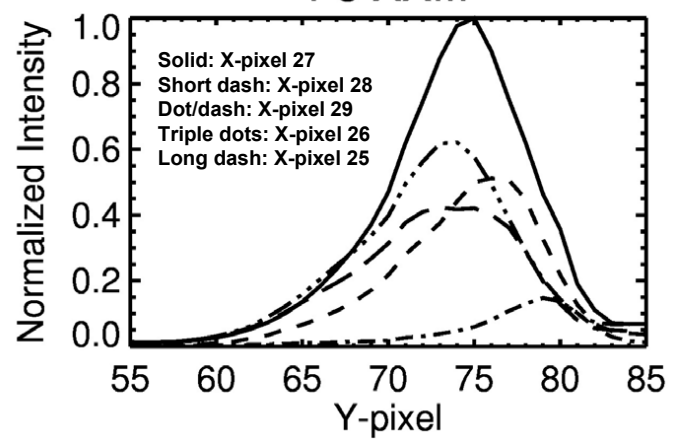

Fe XIII

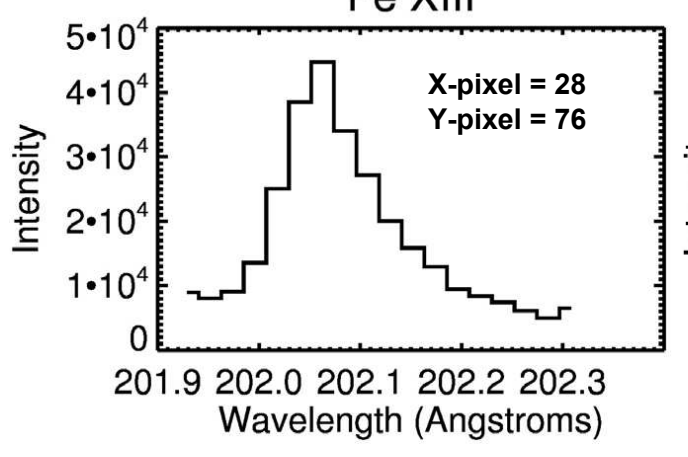

Fe XXIII

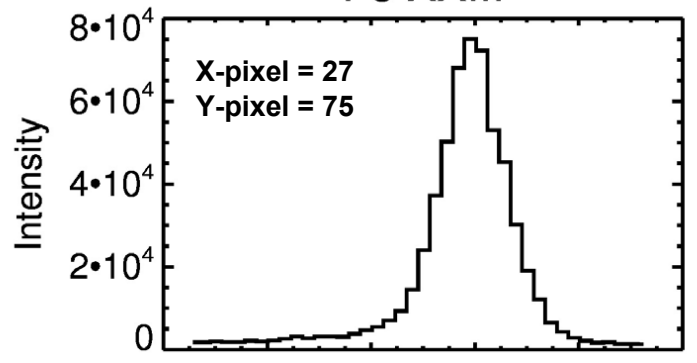

263.4263 .6263 .8264 .0264 .2

Wavelength (Angstroms)

Fe XIV

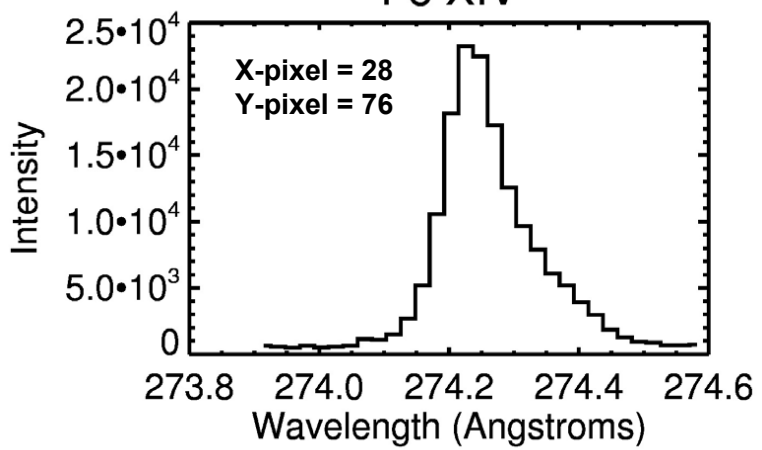

Fig. 15.- Fe XXIII line intensities and selected line profiles for the indicated lines around position $(27,75)$, a location close to, or at, a significant flare energy input. 

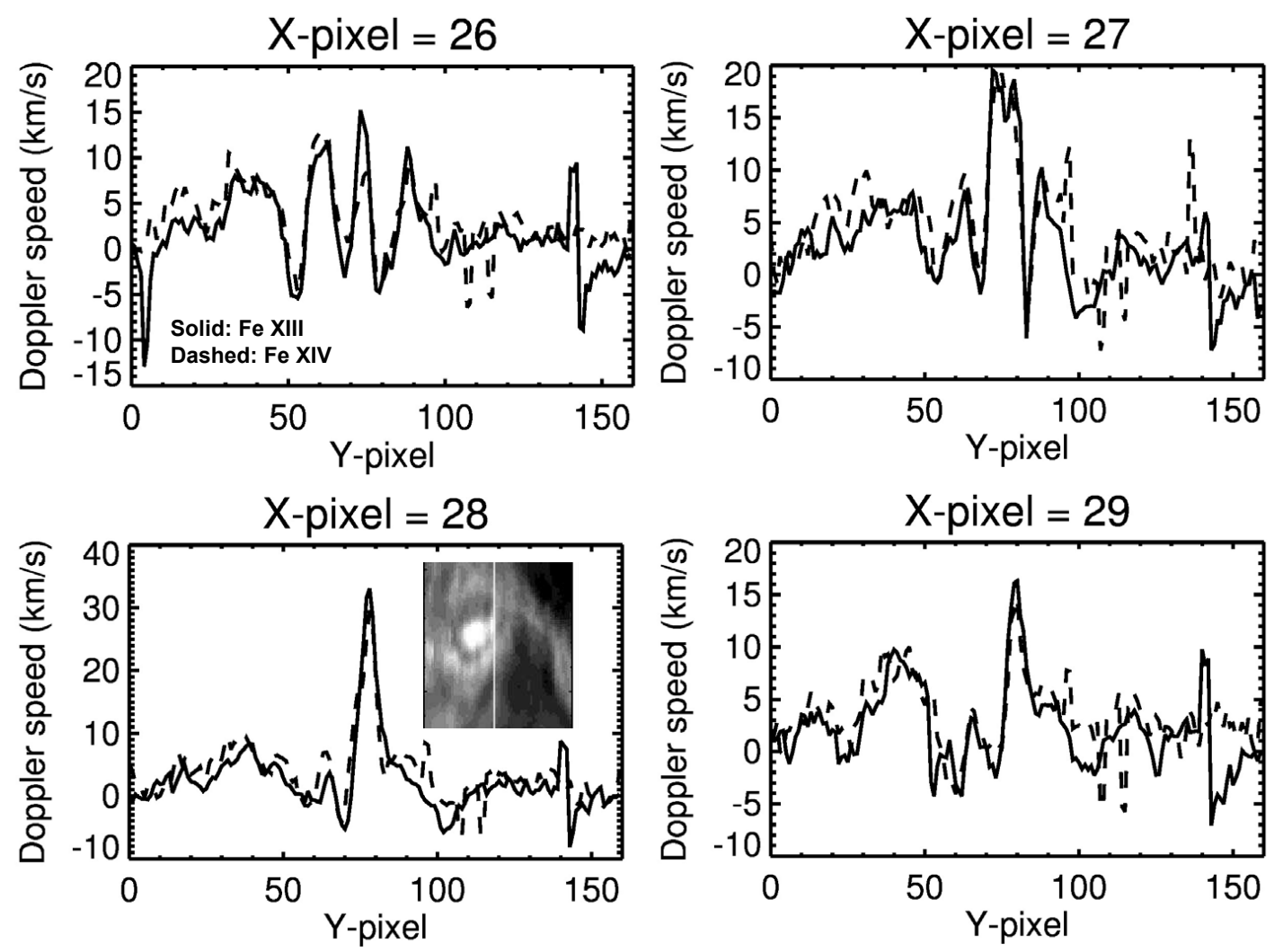

Fig. 16.- Doppler speeds for the indicated lines at four selected X-pixels for all Y-pixels associated with them. 


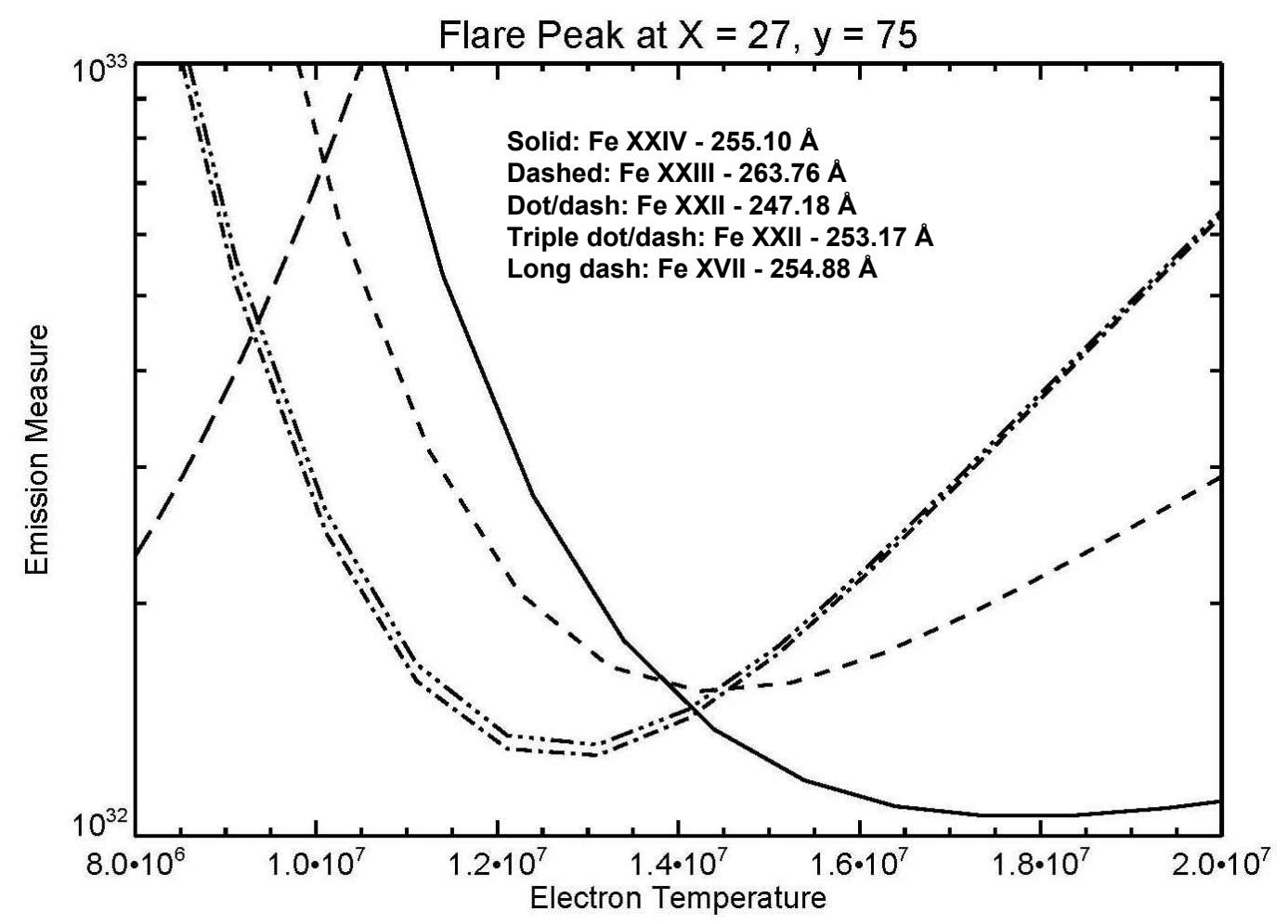

Fig. 17.- Loci plots for the lines shown assuming ionization equilibrium. Except for the lowtemperature Fe XVII line, the curves cross near a common point and indicate an isothermal source at a temperature of about $13.7 \mathrm{MK}$. 